\title{
Involvement of STAT3 Signaling in High Glucose-Induced Epithelial Mesenchymal Transition in Human Peritoneal Mesothelial Cell Line HMrSV5
}

\author{
Pei Zhang ${ }^{a}$ Hong Dai $^{a} \quad$ Lei Peng $^{b}$ \\ a Department of Nephrology, The First Affiliated Hospital of Anhui Medical University, Hefei, \\ China; ${ }^{b}$ Institute of Pharmacology, Anhui Medical University, Hefei, China
}

\section{Keywords}

Peritoneal fibrosis · Glucose · Epithelial mesenchymal transition · STAT3

\begin{abstract}
Background/Aims: Peritoneal fibrosis (PF) is a common complication in patients receiving long-term peritoneal dialysis, which results in damage to peritoneal functions. Epithelial-mesenchymal transition (EMT) is a key step in the early pathogenesis of PF. Increasing evidence has shown that signal transducer and activator of transcription 3 (STAT3) signaling pathway is involved in EMT and tissue fibrosis by interacting with distinct EMT-inducing molecules, including transforming growth factor (TGF)- $\beta$ and advanced glycation end products (AGEs). This study investigated the involvement of STAT3 in the PF process. Methods: We used high glucose-treated human peritoneal mesothelial cell line HMrSV5 as an in vitro model to expose the peritoneal mesothelial cells to high-glucose dialysate. Expression of EMT markers was detected by qRT-PCR. Accumulation of methylglyoxal (MGO) and AGEs in the culture supernatant were measured by enzyme-linked immunosorbent assay. Phosphorylation of STAT3 was assessed by Western blot. Results: Results showed that high glucose upregulated TGF- $\beta$, increased the productions of MGO and AGEs, and induced EMT in HMrSV5 cells. High glucose also activated the STAT3 pathway. STAT3 inhibitor reduced the high glucose-induced EMT, via reducing TGF- $\beta$ expression and repressing the accumulation of MGO and AGEs. Conclusion: Our results revealed a critical role for STAT3 signaling in high glucose-induced EMT in HMrSV5 cells, and suggested that inhibition of STAT3 might be a treatment for high glucose-induced fibrogenesis in PF.




\section{Kidney \\ Blood Pressure \\ Research}

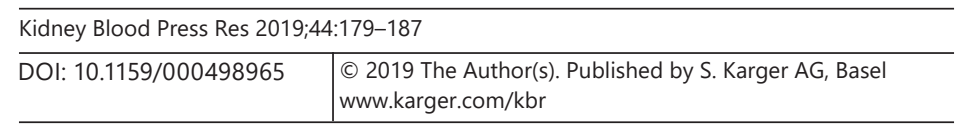

Zhang et al.: Involvement of STAT3 Signaling in HMrSV5 Cells

\section{Introduction}

Peritoneal dialysis (PD) is a well-established renal replacement therapy against endstage renal diseases. In this process the peritoneum of the patient is used as the membrane, through which solutes and fluid are exchanged between the dialysate and the blood. Glucose solution is commonly used as the osmotic agent of the dialysate. However, due to the high glucose concentration, dialysate is bio-incompatible [1]. Long-term use of high-glucose dialysate could induce epithelial-mesenchymal transition (EMT) in peritoneal mesothelial cells, leading to peritoneal fibrosis (PF) [2], which reduces dialysis efficacy and ultimately makes patients withdraw from PD treatment.

One mechanism underlying the high glucose dialysate-induced PF is the accumulation of methylglyoxal (MGO) and advanced glycation end products (AGEs). MGO is a byproduct of glycolysis formed mainly from the degradation of triose phosphates, a tiny "leak" from the glycolysis process [3]. Previous study found that peritoneal injection of MGO accelerated the conventional PD dialysate-induced progression of fibrous peritoneal thickening [4]. MGO was identified as the major precursor of AGEs [5, 6]. Accumulation of AGEs in the interstitium and vessel walls was found to associate with interstitial fibrosis in patients receiving long-term PD [7]. However, mechanism underlying PF induction by MGO and AGEs is not fully studied.

It has been well documented that the signal transducer and activator of transcription 3 (STAT3) signaling pathways are involved in EMT and tissue fibrosis [8]. Activation of STAT3 could induce the expression of the EMT regulator gene SNAIL and thus promote EMT $[9,10]$. Previous study showed that the Janus Kinase (JAK)/STAT3 pathway was essential in transforming growth factor (TGF)- $\beta$-induced EMT of human lung cancer cell lines [11]. Inhibiting STAT3 activity by paclitaxel was found to attenuate renal interstitial fibrosis in mice with ureteral obstruction [12]. A recent study found that in mouse podocytes, AGEs could activate the STAT3 pathway and exert pro-apoptotic and pro-inflammatory effects [13]. However, it is unclear whether STAT3 pathway is also involved in AGEs mediated PF.

This study aimed to investigate the involvement of STAT3 pathway in the PF process using high glucose-treated human peritoneal mesothelial cells HMrSV5 as an in vitro model.

\section{Materials and Methods}

\section{Cell Culture and Treatment}

HMrSV5 cells were purchased from Jennio Biotech Co., Ltd. (Guangzhou, China) and maintained in Dulbecco's Modified Eagle's medium (Gibco, Grand Island, NY, USA) containing 10\% fetal bovine serum (Gibco), $100 \mu \mathrm{g} / \mathrm{mL}$ streptomycin and $100 \mathrm{U} / \mathrm{mL}$ penicillin. For high glucose treatment, cells were first seeded into cell culture plates. After the cells were attached to the culture plates, cells were treated with phosphate-buffered saline (PBS) or $4.25 \%$ glucose for increasing periods of time $(24,48$ or $72 \mathrm{~h}$ ), respectively. To study the role of STAT3 under high glucose condition, cells were co-treated with STAT3 inhibitor BP-1-102 (5 $\mu \mathrm{M}$, purchased from Selleck, Shanghai, China) and $4.25 \%$ glucose for $72 \mathrm{~h}$. Then cells were harvested for further experiments.

\section{qRT-PCR}

Total RNA was purified by Trizol method (Invitrogen, Waltham, MA, USA). Quantitative RT-PCR was carried out using One Step TB Green PrimeScript Kit (Taraka, Dalian, China). The relative mRNA amount of target gene was normalized to GAPDH, using the $2^{-\Delta \Delta C \mathrm{Ct}}$ method. The primers were as follows: vimentin: 5' -TTGAACGCAAAGTGGAATC- $3^{\prime}$ and $5^{\prime}$-AGGTCAGGCTTGGAAACA-3'; N-cadherin, 5'-CGAGCCGCCTGCGCTGCCAC-3' and 5'-C GCTGCTCTCCGCTCCCCGC-3'; TGF- $\beta$ : $5^{\prime}$-AGCAACAATTCCTGGCGATAC-3' and $5^{\prime}$-CAACCACTGCCGCACAACT-3'; 


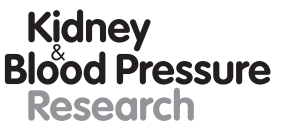

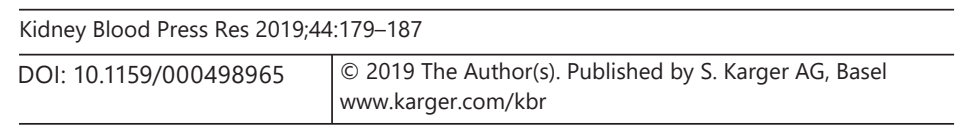

Zhang et al.: Involvement of STAT3 Signaling in HMrSV5 Cells

fibronectin (FN): 5'-CGGTGGCTGTCAGTCAAAG-3' and 5'-AAACCTCGGCTTCCTCCATAA-3'; $\alpha$-SMA: $5^{\prime}$-CCCTTGAGAAGAGTTACGAGTTG- ${ }^{\prime}$ and $5^{\prime}$-TGATGCTGTTGTAGGTGGTTTC- $3^{\prime}$; Ecadherin: $5^{\prime}$-GTCACTGACACCAACGATATTCCT- $3^{\prime}$ and $5^{\prime}$-TTTCAGTGTGGTGATTACGACGTT3'; GAPDH: 5'-ACACCCACTCCTCCACCTTT-3' and 5'-TTACTCCTTGGAGGCCATGT-3'.

\section{Assessment of Cell Proliferation and Apoptosis}

Cells were treated with $4.25 \%$ glucose for increasing periods of time $(24,48$ or $72 \mathrm{~h})$, respectively. Then cell apoptosis was measured with an Annexin-V-FITC/PI apoptosis kit (KeyGEN Biotech, Nanjing, China), following by detection using flow cytometric analysis (BD Biosciences, San Jose, CA, USA). Cell proliferation was measured by Cell Counting Kit-8 (CCK-8) assay using a commercial kit (Kumamoto, Japan) following the manual.

\section{Measurement of MGO and AGEs}

Cells were treated with $4.25 \%$ glucose for increasing periods of time $(24,48$ or $72 \mathrm{~h})$, respectively. Then the supernatants were collected, and the concentrations of MGO and AGEs were measured by enzyme-linked immunosorbent assay (ELISA), according to the manuals (kits for MGO ELISA were purchased from HycultBiotech, Netherlands; kits for AGEs ELISA were purchased from Cusabio, Wuhan, China).

\section{Western Blot}

Cells were lysed using RIPA buffer. Total protein was extracted and separated by SDS-PAGE. Western blot was carried out using standard method [14]. The primary antibodies were: anti-STAT3 (1:1,000, Cell Signaling Technology, Danvers, MA, USA), anti-phos-STAT3 (Tyr-705) (1:1,000, Cell Signaling Technology) and anti- $\beta$-actin (as control, 1:2,000, Abcam).

\section{Statistics}

Data were presented as mean \pm standard deviation (SD). One-way ANOVA followed by post hoc Tukey's test was carried out to evaluate the differences among groups. $t$ test was used to compare the difference between two groups. $p<0.05$ was considered statistically significant.

\section{Results}

High Glucose Induces EMT in HMrSV5 Cells

We measured the mRNA levels of several EMT markers, including mesenchymal markers ( $\alpha$-SMA, etc.) and epithelial-specific marker (E-cadherin), to determine the extent of EMT in high glucose-treated HMrSV5 cells. As shown in Figure 1, compared with PBS-treated cells, the mRNA levels of vimentin $(2.639 \pm 0.377)$, N-cadherin $(4.367 \pm 0.707)$, TGF- $\beta(1.929 \pm$ $0.103)$, FN $(3.813 \pm 0.516)$ and $\alpha$-SMA (3.932 \pm 0.630$)$ were significantly elevated in high glucose-treated cells, while E-cadherin $(0.588 \pm 0.083) \mathrm{mRNA}$ expression was significantly reduced. These results indicated that high glucose could induce EMT in HMrSV5 cells.

\section{Effect of High Glucose on Apoptosis and Proliferation of HMrSV5 Cells}

As shown in Figure 2, the percentage of Annexin-V and PI double positive cells (apoptotic cells) was not influenced by high glucose treatment at any time point $(7.97 \pm 0.17 \%, 7.79 \pm$ $0.64 \%, 7.84 \pm 0.29 \%, 7.71 \pm 0.20 \%$ ) (Fig. $2 \mathrm{a}$ and $b$ ), showing that high glucose had no effect on cell apoptosis. On the other hand, results of CCK-8 assays showed that the viability of HMrSV 5 cells $(0.949 \pm 0.039)$ was significantly reduced after $48 \mathrm{~h}$ of high glucose treatment $(0.808 \pm 0.041)$, and the cell viability was further reduced after $72 \mathrm{~h}$ treatment $(0.521 \pm 0.053)$ (Fig. 2c), indicating that high glucose inhibited proliferation of HMrSV5 cells. 
Kidney

Blood Pressure

Research

Fig. 1. High glucose-induced epithelial to mesenchymal transformation of Human peritoneal mesothelial cells HMrSV5. The mRNA level of vimentin, $\mathrm{N}$-cadherin, TGF- $\beta, F N, \alpha$-SMA, and E-cadherin in HMrSV 5 cells treated with PBS or $4.25 \%$ glucose for $72 \mathrm{~h}$ were determined by qRTPCR. Data were presented by mean \pm SD, $n=3$ independent experiments. ${ }^{*} p<0.05$, ** $p<0.01$, and ${ }^{* * *} p<0.001$ compared to PBS group.

\begin{tabular}{l|l}
\hline Kidney Blood Press Res 2019;44:179-187 \\
\hline DOI: 10.1159/000498965 & $\begin{array}{l}\text { @ 2019 The Author(s). Published by S. Karger AG, Basel } \\
\text { www.karger.com/kbr }\end{array}$ \\
\hline
\end{tabular}
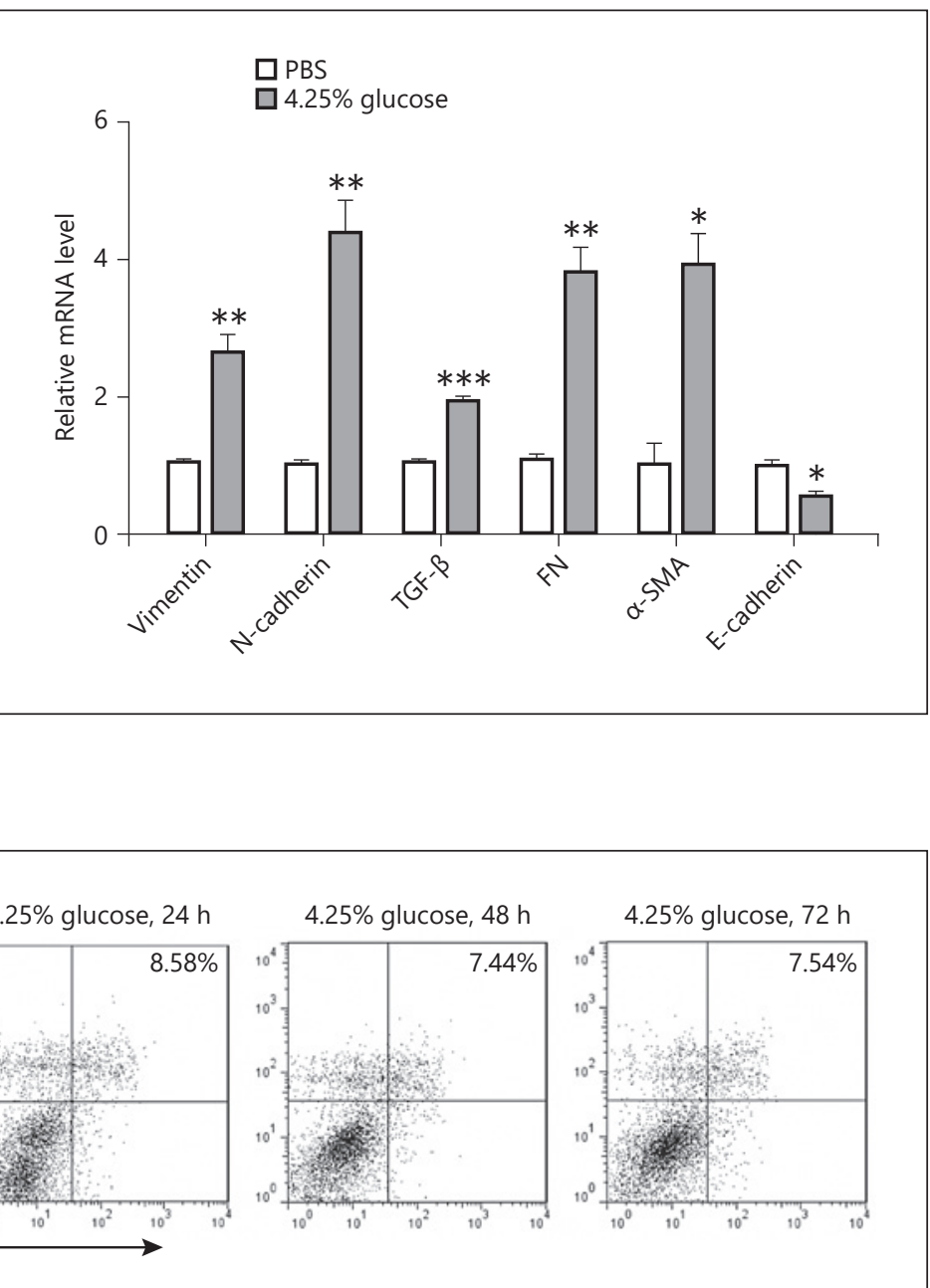

a
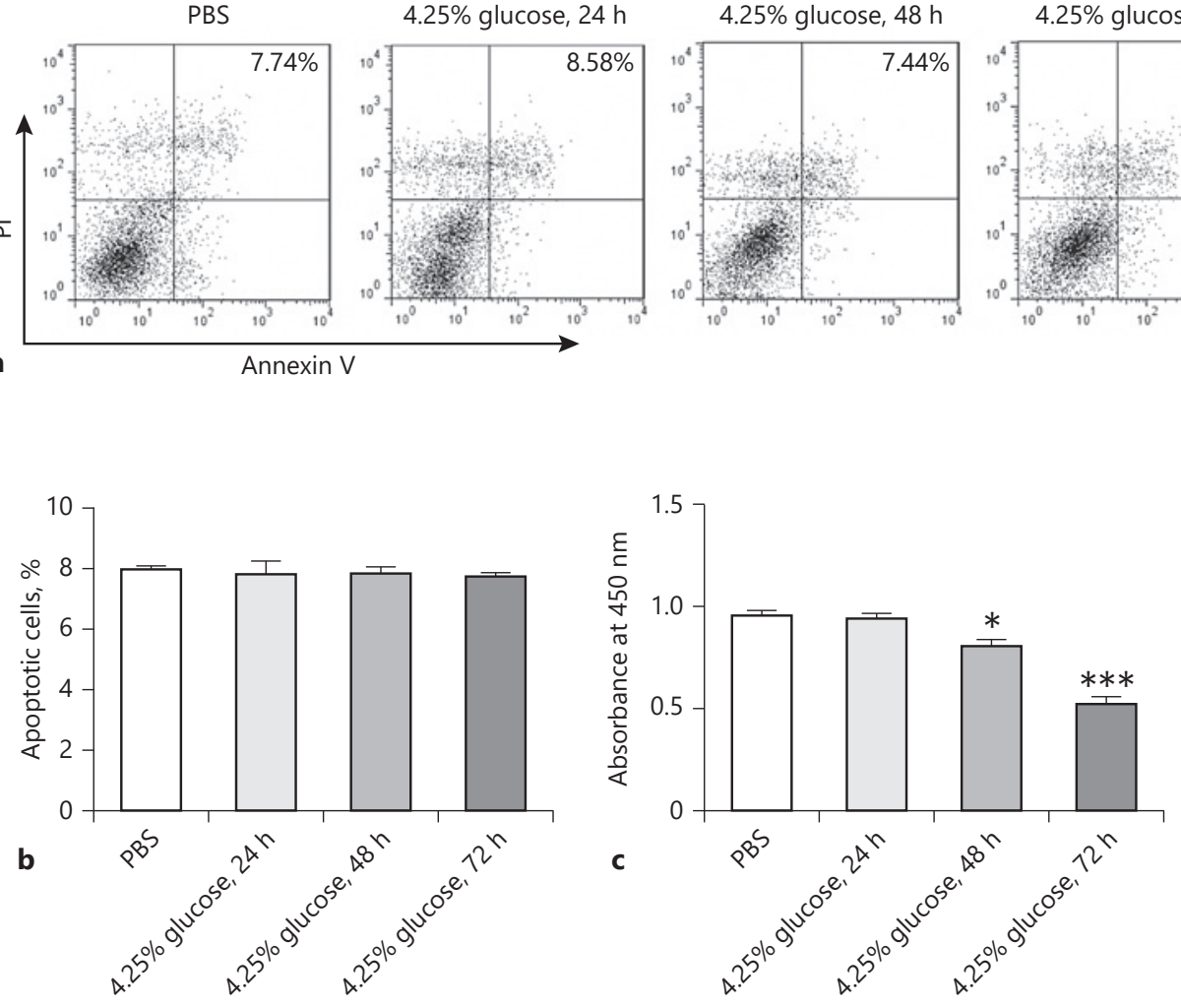

Fig. 2. Effect of high glucose on apoptosis and proliferation of HMrSV5 cells. a The apoptosis of HMrSV5 cells treated with $4.25 \%$ glucose for 24, 48, or $72 \mathrm{~h}$ were determined by Annexin V-FITC \& PI staining kit through flow cytometry. b Statistical data of panel a. Data showed the Annexin V and PI double positive cells. Data were represented as mean $\pm \mathrm{SD}, n=3$ independent experiments. $\mathbf{c}$ The viability of HMrSV 5 cells treated with $4.25 \%$ glucose for 24,48 , or $72 \mathrm{~h}$ were determined by cck-8 kit. Data were represented as mean $\pm \mathrm{SD}, n=3$ independent experiments. ${ }^{*} p<0.05$ and ${ }^{* * *} p<0.001$ compared to the PBS group. 


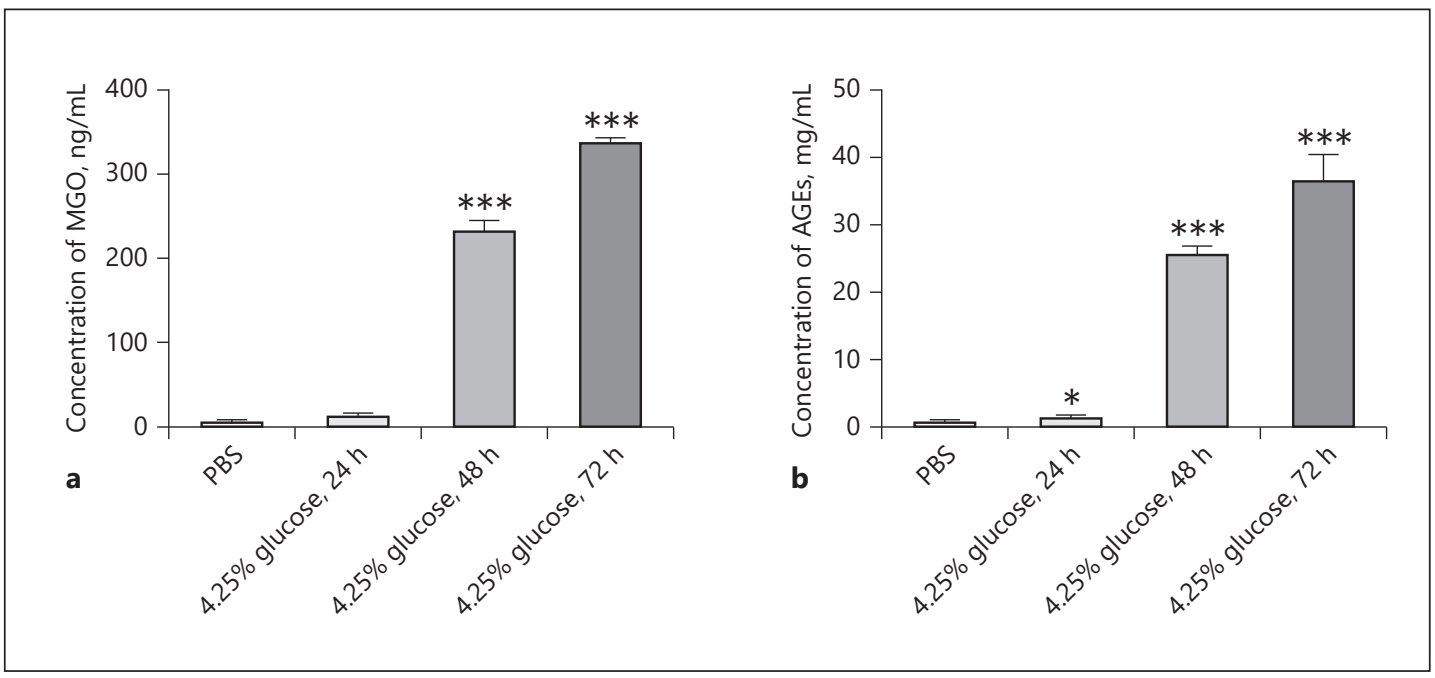

Fig. 3. High glucose induced the accumulation of MGO and AGEs in the supernatants of HMrSV5 cells. HMrSV5 cells were treated with $4.25 \%$ glucose for 24,48 , or $72 \mathrm{~h}$ and the concentration of MGO (a) or AGEs (b) in the supernatants were determined by ELISA. Data were presented by mean \pm SD, $n=3$ independent experiments. $* p<0.05$ and ${ }^{* * *} p<0.001$ compared to the PBS group.

High Glucose Induces Accumulation of MGO and AGEs in the Supernatant of HMrSV5 Cells

MGO is a byproduct of glycolysis and the major precursor of AGEs, which play an important role in EMT. Here we found that the concentrations of MGO and AGEs in the supernatant of high glucose treated cells were significantly increased compared with PBS-treated cells, in a time-dependent manner (Fig. 3a and b), indicating that high glucose induced production of MGO and AGEs in HMrSV5 cells.

\section{High Glucose Upregulates STAT3 and phos-STAT3 in HMrSV5 Cells}

Results of Western blot showed that the protein levels of STAT3 and phosphorylated STAT3 (Tyr-705) in the cells were significantly increased after $48 \mathrm{~h}$ of high glucose treatment, which were further increased after $72 \mathrm{~h}$ treatment (Fig. 4a-c), compared with PBS-treated cells. These results showed that high glucose could activate STAT3 signaling in HMrSV5 cells.

\section{STAT3 Inhibitor Reverses the Effect of High Glucose in HMrSV5 Cells}

As shown in Figure 5, compared with high glucose-treated cells, cells co-treated with STAT3 inhibitor BP-1-102 showed decreased mesenchymal markers (vimentin, $\alpha$-SMA, etc.) and increased epithelial-specific marker (E-cadherin) (Fig. 5a), increased cell viability (Fig. 5b), and decreased concentrations of MGO and AGEs in the cell supernatant (Fig. 5c and d). These results indicated that BP-1-102 could reverse the effect of high glucose in HMrSV5 cells.

\section{Discussion}

In this study we sought to investigate the involvement of STAT3 pathway in the PF process. We used high glucose-treated human peritoneal mesothelial cell line HMrSV5 as an in vitro model to expose the peritoneal mesothelial cells to high-glucose dialysate. As expected, we found that high glucose induced EMT in HMrSV5 cells. It was well documented that EMT 


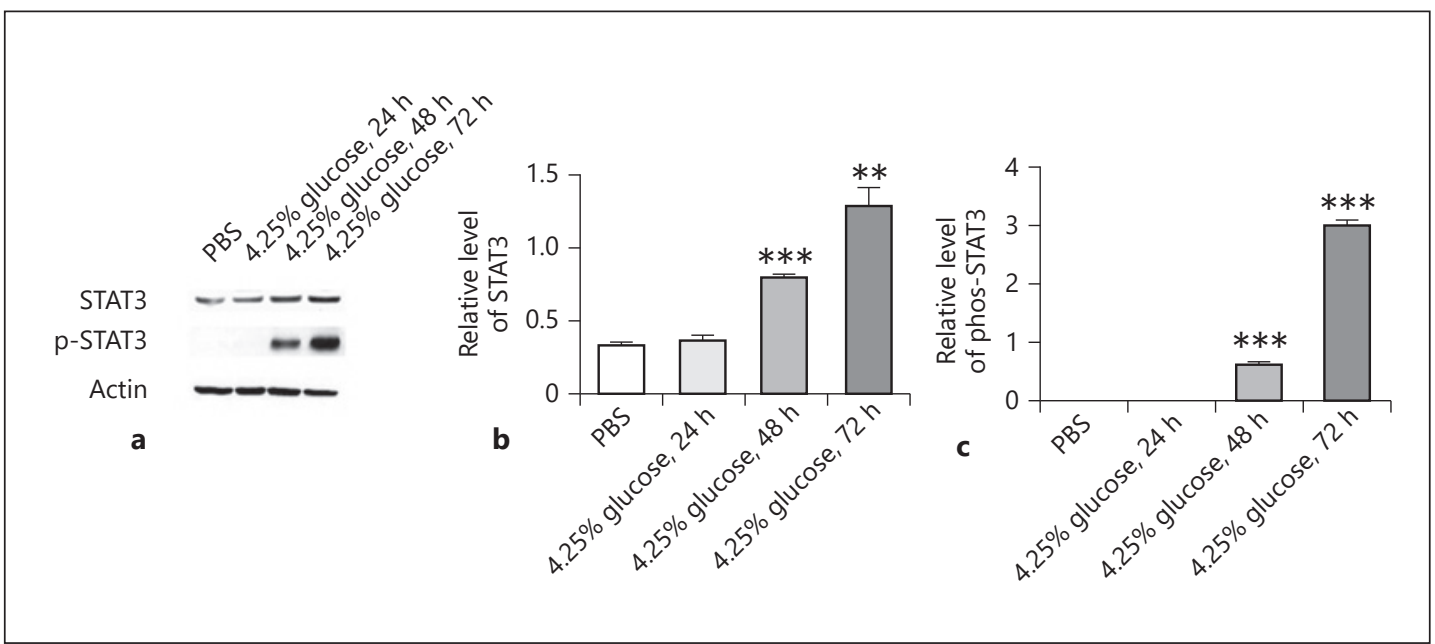

Fig. 4. High glucose upregulated STAT3 and phos-STAT3 in HMrSV5 cells. a Western blot showing the protein expression level of STAT3 and phos-STAT3 (Tyr-705) in the treatment of $4.25 \%$ glucose for 24,48 , or 72 h. $N=3$ independent experiments and this panel presented one of these repeats. $\mathbf{b}$ Statistic of the relative protein level of STAT3 in panel a. Data were presented by mean \pm SD. $N=3$ independent experiments. ${ }^{* *} p<$ 0.01 and ${ }^{* * *} p<0.001$ compared to the PBS group. c Statistic of the relative level of phos-STAT3 (Tyr-705) in panel a. Data were presented by mean $\pm \mathrm{SD}, n=3$ independent experiments. ${ }^{* * *} p<0.001$ compared to the PBS group.

was the key point in the early pathogenesis of PF associated with PD [15]. Many distinct molecular changes occur during the EMT process, including transcriptional factor activation, expression of specific cell-surface proteins, etc. [16]. Some molecules during this process are used as biomarkers to indicate EMT extent in cells. For example, E-cadherin is an epithelial cell adhesion molecule, and its expression was found to be decreased during EMT in tissue fibrosis [17]. N-cadherin is expressed in mesenchymal cells, and cadherin switch (changes in the expression level) from E-cadherin to N-cadherin has been used to monitor EMT [18]. Upregulated $\alpha$-SMA and vimentin are classic markers of EMT, and have been used to identify cells in the midst of EMT $[15,16]$. FN is a component of extracellular matrix that is associated with tissue fibrosis, and was found to be upregulated during EMT [15]. In this study, we found that the expression pattern of these molecules in high glucose-treated HMrSV5 cells was in accordance with that observed in EMT, demonstrating that high glucose induced EMT in HMrSV5 cells. Meanwhile, we found that the cell proliferation was significantly reduced after treatment with high glucose. This result might be attributed to the conversion of cells from mesothelial cells to fibroblasts, thus resulting in reduced proliferation.

TGF- $\beta$ plays a critical role in PF through inducing EMT. It could downregulate E-cadherin expression via the SNAIL pathway, and induce epithelial cells to lose cell-cell contact [19]. Inhibitor of TGF- $\beta$, BMP-7, was found to reverse the TGF- $\beta$-induced downregulation of E-cadherin. In vivo data showed that BMP-7 reversed EMT and repaired the damage of epithelial structures in mouse models of renal injury and fibrosis [20]. Previous study found that high glucose could increase TGF- $\beta$ activity through Smad 2 acetylation [21]. Moreover, high glucose might also stimulate the synthesis of TGF- $\beta$ through the formation of AGEs [15, $22]$. AGEs are mainly formed from MGO, a byproduct of glycolysis, and are found to play an important role in EMT [23]. Studies showed that AGEs could bind to the AGE receptors on the cell membrane of rat kidney epithelial cells, and induce the expression of TGF- $\beta$ which promoted EMT [24]. In this study we found that high glucose treatment resulted in increased 


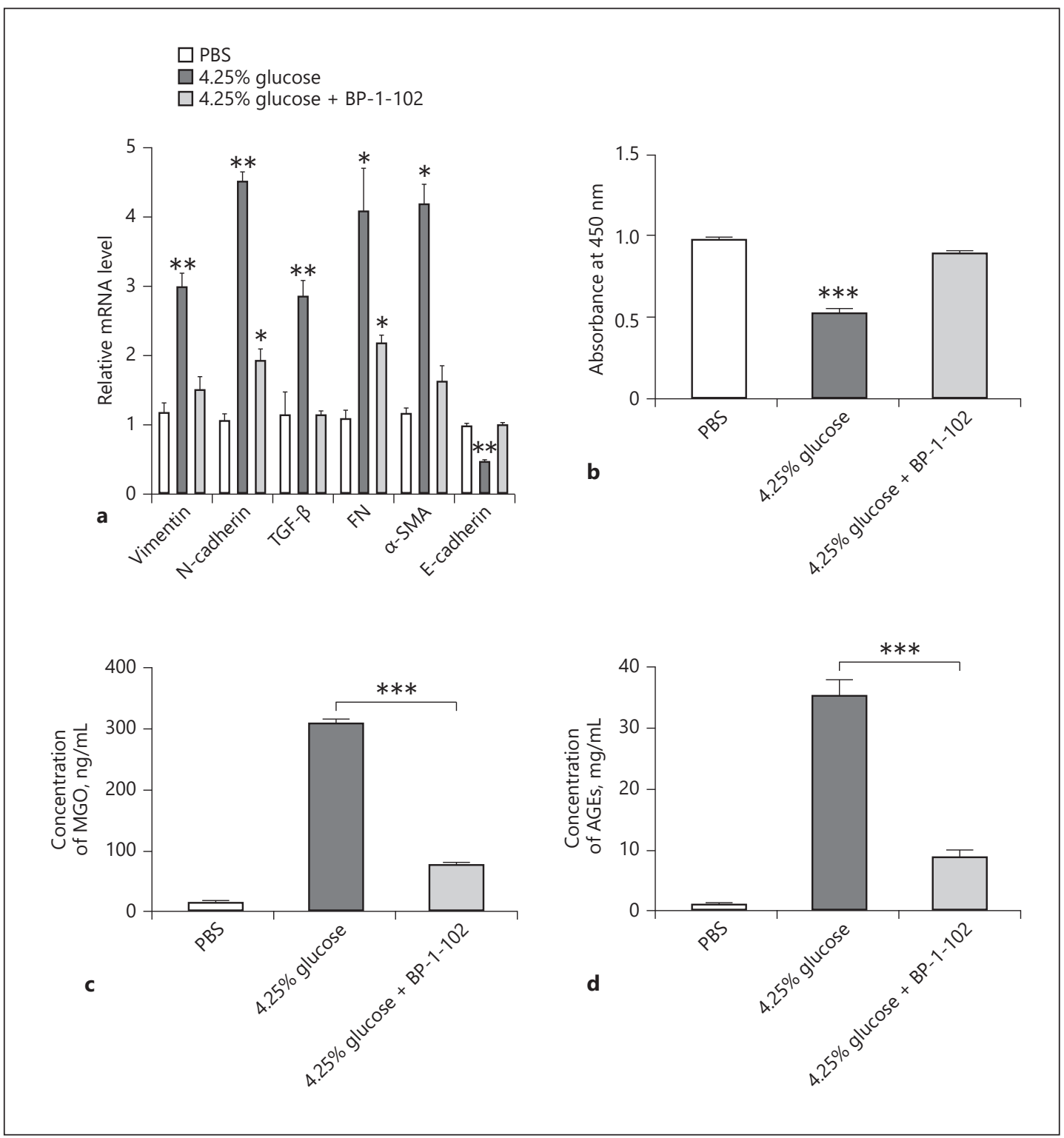

Fig. 5. The inhibitor of STAT3 restored the glycolysis to the normal situation. a The mRNA level of vimentin, $\mathrm{N}$-cadherin, TGF- $\beta$, FN, $\alpha$-SMA, and E-cadherin in HMrSV 5 cells treated with $4.25 \%$ glucose for $72 \mathrm{~h}$ in the presence or absence of STAT3 inhibitor BP-1-102 were determined by qRT-PCR. Data were presented by mean \pm SD, $n=3$ independent experiments. ${ }^{*} p<0.05,{ }^{* *} p<0.01$, and ${ }^{*} p>0.05$ compared to PBS group. $\mathbf{b}$ The viability of HMrSV 5 cells treated with $4.25 \%$ glucose for $72 \mathrm{~h}$ in the presence or absence of STAT3 inhibitor BP-1-102 were determined by cck-8 kit. Data were represented as mean $\pm \mathrm{SD}, n=3$ independent experiments. ${ }^{* * *} p<$ 0.001 compared to the PBS group. c, $\mathbf{d}$ HMrSV5 cells were treated with $4.25 \%$ glucose for $72 \mathrm{~h}$ in the presence or absence of STAT3 inhibitor BP-1-102 and the concentration of MGO (c) or AGEs (d) in the supernatants were determined by ELISA. Data were presented by mean \pm SD, $n=3$ independent experiments. ${ }^{* * *} p<0.001$.

TGF- $\beta$ expression, along with increased MGO and AGEs concentrations, demonstrating that high glucose could promote EMT via inducing AGEs and TGF- $\beta$.

Increasing evidence has shown that the STAT3 signaling pathways are involved in EMT and tissue fibrosis. STAT3 is a transcription factor, whose transcriptional activity is positively regulated by phosphorylation at the Tyr705 residue by JAKs [25]. Previous study showed that 


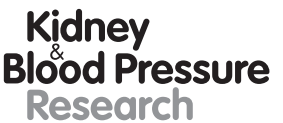

\begin{tabular}{l|l}
\hline Kidney Blood Press Res 2019;44:179-187 \\
\hline DOI: 10.1159/000498965 & $\begin{array}{l}\text { @ 2019 The Author(s). Published by S. Karger AG, Basel } \\
\text { www.karger.com/kbr }\end{array}$ \\
\hline
\end{tabular}

Zhang et al.: Involvement of STAT3 Signaling in HMrSV5 Cells

the activation of JAK/STAT3 pathway was required in TGF- $\beta$-induced EMT of human lung cancer cell lines [11]. Activation of JAK/STAT3 pathway was also associated with liver fibrosis in mouse models [26]. Upregulation of phosphorylated STAT3 at Tyr705 was observed in a rat model of renal fibrosis [27]. In accordance with these results, we found that high glucose treatment resulted in upregulation of phosphorylated STAT3 at Tyr705 in HMrSV5 cells, indicating that high glucose induced EMT in these cells via activating the STAT3 pathway. To further verify the involvement of STAT3 in this process, we examined the effect of STAT3 inhibition in high glucose-induced EMT. BP-1-102 is a phospho-tyrosine mimic that could bind the STAT3-SH2 domain and thus block the phosphorylation of STAT3 at Tyr residues [28]. We found that BP-1-102 reduced the expression of TGF- $\beta$ and suppressed EMT after high glucose treatment. Our results were consistent with a previous study, which showed that treatment with S3I-201 (another STAT3 inhibitor that diminishes STAT3 tyrosine phosphorylation) inhibited the expression of $\alpha$-SMA, reduced the deposition of extracellular matrix proteins and attenuated renal fibrosis [25]. It is interesting that in this study, we found BP-1-102 reduced the concentrations of MGO and AGEs after high glucose treatment. It was well documented that AGE could induce the activation of the STAT3 pathway to exert biological functions $[13,29]$. However, the mechanism responsible for STAT3 pathway regulation on the production of MGO and AGEs remains unclear, which requires further investigation.

\section{Conclusions}

In conclusion, our current study demonstrates that high glucose induces EMT in HMrSV5 cells via upregulating TGF- $\beta$ and increasing the production of MGO and AGEs. High glucose also activates the STAT3 pathway. Treatment with STAT3 inhibitor reverses the high glucoseinduced changes in EMT markers and thus reduces EMT, indicating that the effect of high glucose in inducing EMT is mediated by the STAT3 pathway.

\section{Acknowledgments}

The study was supported by the Nature Science Foundation of Anhui Province (1808085MH249).

\section{Disclosure Statement}

The authors declare that there is no conflict of interest.

\section{References}

1 de Lima SM, Otoni A, Sabino AP, Dusse LM, Gomes KB, Pinto SW, et al. Inflammation, neoangiogenesis and fibrosis in peritoneal dialysis. Clin Chim Acta. 2013 Jun;421:46-50.

2 Zhou Q, Bajo MA, Del Peso G, Yu X, Selgas R. Preventing peritoneal membrane fibrosis in peritoneal dialysis patients. Kidney Int. 2016 Sep; 90(3):515-24.

3 Rabbani N, Thornalley PJ. The critical role of methylglyoxal and glyoxalase 1 in diabetic nephropathy. Diabetes. 2014 Jan;63(1):50-2.

4 Onishi A, Akimoto T, Morishita Y, Hirahara I, Inoue M, Kusano E, Nagata D: Peritoneal fibrosis induced by intraperitoneal methylglyoxal injection: The role of concurrent renal dysfunction. Am J Nephrol. 2014;40(4):38190.

5 Maessen DE, Stehouwer CD, Schalkwijk CG. The role of methylglyoxal and the glyoxalase system in diabetes and other age-related diseases. Clin Sci (Lond). 2015 Jun;128(12):839-61. 
6 Schalkwijk CG. Vascular AGE-ing by methylglyoxal: the past, the present and the future. Diabetologia. 2015 Aug;58(8):1715-9.

7 Honda K, Nitta K, Horita S, Yumura W, Nihei H, Nagai R, Ikeda K, Horiuchi S: Accumulation of advanced glycation end products in the peritoneal vasculature of continuous ambulatory peritoneal dialysis patients with low ultra-filtration. Nephrol Dial Transplant. 1999 Jun;14(6):1541-9.

8 Lamouille S, Xu J, Derynck R. Molecular mechanisms of epithelial-mesenchymal transition. Nat Rev Mol Cell Biol. 2014 Mar;15(3):178-96.

9 Xiao X, Fischbach S, Zhang T, Chen C, Sheng Q, Zimmerman R, et al. Smad3/stat3 signaling mediates $\beta$-cell epithelial-mesenchymal transition in chronic pancreatitis-related diabetes. Diabetes. 2017 Oct;66(10):264658.

10 Yadav A, Kumar B, Datta J, Teknos TN, Kumar P. IL-6 promotes head and neck tumor metastasis by inducing epithelial-mesenchymal transition via the JAK-STAT3-SNAIL signaling pathway. Mol Cancer Res. 2011 Dec; 9(12):1658-67.

11 Liu R, Zeng Y, Lei Z, Wang L, Yang H, Liu Z, Jun Z, Zhang H-T: JAK/STAT3 signaling is required for TGF- $\beta$ induced epithelial-mesenchymal transition in lung cancer cells. Int J Oncol. 2014 May;44(5):1643-51.

12 Zhang L, Xu X, Yang R, Chen J, Wang S, Yang J, et al. Paclitaxel attenuates renal interstitial fibroblast activation and interstitial fibrosis by inhibiting STAT3 signaling. Drug Des Devel Ther. 2015 Apr;9:2139-48.

$13 \mathrm{Yu} \mathrm{J}$, Wu H, Liu ZY, Zhu Q, Shan C, Zhang KQ. Advanced glycation end products induce the apoptosis of and inflammation in mouse podocytes through CXCL9-mediated JAK2/STAT3 pathway activation. Int J Mol Med. 2017 Oct;40(4):1185-93.

14 Bernier M, Paul RK, Martin-Montalvo A, Scheibye-Knudsen M, Song S, He HJ, et al. Negative regulation of STAT3 protein-mediated cellular respiration by SIRT1 protein. J Biol Chem. 2011 Jun;286(22):19270-9.

15 Aroeira LS, Aguilera A, Sánchez-Tomero JA, Bajo MA, del Peso G, Jiménez-Heffernan JA, et al. Epithelial to mesenchymal transition and peritoneal membrane failure in peritoneal dialysis patients: pathologic significance and potential therapeutic interventions. J Am Soc Nephrol. 2007 Jul;18(7):2004-13.

16 Kalluri R, Weinberg RA. The basics of epithelial-mesenchymal transition. J Clin Invest. 2009 Jun;119(6): 1420-8.

17 Hay ED, Zuk A. Transformations between epithelium and mesenchyme: normal, pathological, and experimentally induced. Am J Kidney Dis. 1995 Oct;26(4):678-90.

18 Zeisberg M, Neilson EG. Biomarkers for epithelial-mesenchymal transitions. J Clin Invest. 2009 Jun;119(6): 1429-37.

19 Yu H, Shen Y, Hong J, Xia Q, Zhou F, Liu X. The contribution of TGF- $\beta$ in Epithelial-Mesenchymal Transition (EMT): down-regulation of E-cadherin via snail. Neoplasma. 2015;62(1):1-15.

20 Zeisberg M, Bottiglio C, Kumar N, Maeshima Y, Strutz F, Müller GA, et al. Bone morphogenic protein-7 inhibits progression of chronic renal fibrosis associated with two genetic mouse models. Am J Physiol Renal Physiol. 2003 Dec;285(6):F1060-7.

21 Bugyei-Twum A, Advani A, Advani SL, Zhang Y, Thai K, Kelly DJ, et al. High glucose induces Smad activation via the transcriptional coregulator p300 and contributes to cardiac fibrosis and hypertrophy. Cardiovasc Diabetol. 2014 May;13(1):89.

22 Forbes JM, Cooper ME, Oldfield MD, Thomas MC. Role of advanced glycation end products in diabetic nephropathy. J Am Soc Nephrol. 2003 Aug;14(8 Suppl 3):S254-8.

23 Antognelli C, Gambelunghe A, Muzi G, Talesa VN. Glyoxalase I drives epithelial-to-mesenchymal transition via argpyrimidine-modified Hsp70, miR-21 and SMAD signalling in human bronchial cells BEAS-2B chronically exposed to crystalline silica Min-U-Sil 5: transformation into a neoplastic-like phenotype. Free Radic Biol Med. 2016 Mar; 92:110-25.

24 Oldfield MD, Bach LA, Forbes JM, Nikolic-Paterson D, McRobert A, Thallas V, et al. Advanced glycation end products cause epithelial-myofibroblast transdifferentiation via the receptor for advanced glycation end products (RAGE). J Clin Invest. 2001 Dec;108(12):1853-63.

25 Li M, Shougang Z. The role of stat 3 in tissue fibrosis. Curr Chem Biol. 2011;5:44-51.

26 Ogata H, Chinen T, Yoshida T, Kinjyo I, Takaesu G, Shiraishi H, Iida M, Kobayashi T, Yoshimura A: Loss of SOCS3 in the liver promotes fibrosis by enhancing STAT3-mediated TGF-beta1 production. Oncogene. 2006 Apr 20; 25(17):2520-30.

27 Kuratsune M, Masaki T, Hirai T, Kiribayashi K, Yokoyama Y, Arakawa T, et al. Signal transducer and activator of transcription 3 involvement in the development of renal interstitial fibrosis after unilateral ureteral obstruction. Nephrology (Carlton). 2007 Dec;12(6):565-71.

28 Croucher DC, Jimenez-Zepeda VH, Page BD, Li ZH, Wei E, Turkson J, et al. Abstract a201: the potent stat3/5 inhibitor, bp-1-102, demonstrates significant antitumor activity against waldenstrom macroglobulinemia. Mol Cancer Ther. 2011;10 Supplement 1:A201-201.

29 Huang JS, Lee YH, Chuang LY, Guh JY, Hwang JY. Cinnamaldehyde and nitric oxide attenuate advanced glycation end products-induced the Jak/STAT signaling in human renal tubular cells. J Cell Biochem. 2015 Jun;116(6): 1028-38. 